\title{
Mitbestimmung und Leiharbeit
}

Leiharbeit ist aus Sicht von Gewerkschaften und Betriebsräten ein heikles Thema. Zwar gilt sie als Möglichkeit des (Wieder)Einstiegs in den Arbeitsmarkt und dient zugleich der Beschäftigungssicherung der Stammbelegschaften; sie bietet in den meisten Fällen jedoch nur prekäre Arbeitsbedingungen, welche auch die Standards für „Normalbeschäftigte“ und die gewerkschaftliche Solidarität zu untergraben drohen. Die rechtlichen Befugnisse von Betriebsräten in den Einsatzbetrieben beim Thema Leiharbeit sind gering und die Interessenvertretung für Leiharbeitsbeschäftigte ist schwierig. Eine qualitative Studie im Organisationsbereich der IG Metall untersuchte Betriebsratspraktiken und Möglichkeiten der Mitbestimmung zum Thema Leiharbeit.

INGRID ARTUS

\section{Einleitung}

Im Dezember 2012 waren rund 822.000 Menschen und damit etwas weniger als $3 \%$ aller sozialversicherungspflichtig Beschäftigten in Leiharbeit tätig. Obwohl ihre Zahl gegenüber dem Höchststand zum Jahreswechsel 2011/12 leicht zurückgegangen ist, lag sie deutlich höher als vor der Wirtschaftskrise 2008/09 und weit über dem Niveau (von ca. $1 \%$ der Gesamtbeschäftigung) vor der Abschaffung diverser gesetzlicher Beschränkungen der Arbeitnehmerüberlassung im Jahr 2003 (Bundesagentur für Arbeit 2013).

Über Art und Ausmaß der wirtschaftlichen und arbeitsmarktpolitischen Effekte von Leiharbeit existiert eine kontroverse Debatte (vgl. Promberger 2007, 2012; Wölfle 2008; Crimmann et al. 2009; Lehmer 2012; Voss/Vitols 2013). Jüngste Studien gehen davon aus, dass etwa die Hälfte der Leiharbeitsstellen „zusätzliche“ Stellen sind; in den übrigen Fällen werden Stammarbeitsplätze durch Leiharbeit verdrängt (Jahn/Weber 2013). Unstrittig sind die erhöhten Prekaritätsrisiken von Leiharbeit. Dies gilt für die reduzierte Beschäftigungssicherheit, die unterdurchschnittliche Entgelthöhe, die häufig erschwerten Arbeitsbedingungen, geringere Fortbildungsmöglichkeiten sowie die niedrigere Arbeitszufriedenheit (Vogel 2004; Brehmer/Seifert 2007; Keller/Seifert 2007). Leiharbeit hat damit häufig deregulierende Wirkungen auf Lohn- und Arbeitsbedingungen. Diese Effekte treffen auch Beschäftigte, die nicht selbst im pre- kären Segment des Arbeitsmarkts tätig sind. Die verstärkte Nutzung von Leiharbeit kann auch in gewerkschaftlich gut organisierten Großbetrieben zu einer „Destabilisierung des Stabilen“ (vgl. Dörre et al. 2004; Brinkmann et al. 2006) führen. Das Nebeneinander von Stammbeschäftigten und Leiharbeitnehmerinnen und Leiharbeitnehmern verursacht häufig Konkurrenzangst und Leistungsdruck. Auch die Solidarität im Fall betrieblicher oder tariflicher Konflikte ist gefährdet.

In jüngerer Zeit gab es deutliche Re-Regulierungsbemühungen des Staates, der Tarifparteien sowie der Rechtsprechung zum Thema Leiharbeit. Die Organisierungskampagne der IG Metall spielte eine wichtige Rolle für die Politisierung des Themas (vgl. IG Metall 2008). 2011 reformierte der Gesetzgeber das Arbeitnehmerüberlassungsgesetz erneut in einigen Punkten und erklärte einen Mindestlohn in der Leiharbeit für allgemeinverbindlich. Vor allem in „tarifstarken“ Branchen gelang es den Gewerkschaften zudem, Branchenzuschläge im Bereich der Leiharbeit zu vereinbaren, die i.d.R. eine stufenweise Anhebung der Entgelte auf bis zu $100 \%$ des Grundlohns vorsehen (vgl. Schwitzer/Schumann 2013). Im kürzlich unterzeichneten Koalitionsvertrag der neuen Bundesregierung ist eine Begrenzung der Einsatzhöchstdauer auf 18 Monate vereinbart sowie „equal pay“, also der Grundsatz „gleicher Lohn für gleichwertige Arbeit" nach neun Monaten Einsatzzeit. Das Bundesarbeitsgericht revidierte 2013 zudem seine bislang anderslautende Rechtsprechung, sodass künftig im 
Entleihbetrieb regelmäßig beschäftigte Leiharbeitnehmer für die Bestimmung der Größe des Betriebsrats zu berücksichtigen sind. Neu ist auch, dass Betriebsräte die Zustimmung zur Einstellung von als Leiharbeitnehmer Beschäftigten verweigern können, wenn deren Einsatz nicht nur als Übergangslösung geplant ist.

Die neue Rechtsprechung unterstreicht den Sachverhalt, dass in der betrieblichen Praxis die Betriebsräte in den Einsatzbetrieben von Leiharbeit eine nicht zu unterschätzende Rolle spielen, obwohl das Betriebsverfassungsgesetz eigentlich vorsieht, dass Leiharbeitnehmer und Leiharbeitnehmerinnen ihr Mitbestimmungsrecht im Verleihbetrieb ausüben. Erst ab einer Einsatzdauer von drei Monaten besitzen sie zusätzlich auch das aktive (aber nicht das passive) Wahlrecht im Entleihbetrieb. In der Praxis erweist sich dieses „doppelte Recht“ auf Mitbestimmung jedoch als halbierte Mitbestimmungsoption (Wassermann/Rudolph 2007; Artus 2012). Nur in sehr wenigen, vor allem den größeren Leiharbeitsfirmen (wie Randstadt, Adecco, Manpower), existiert überhaupt ein Betriebsrat. Aber auch mit Blick auf die Entleihbetriebe haben Studien ergeben: Dort, wo intensiv Leiharbeit genutzt wird, ist die Betriebsratsdichte unterdurchschnittlich (vgl. Bellmann/ Kühl 2007). Und selbst wenn ein Betriebsrat im Einsatzunternehmen existiert, ist nicht sicher, ob dieser sich für

\section{TABELLE 1}

\section{Die Untersuchungsbetriebe}

Angaben in absoluten Zahlen und in Prozent

\begin{tabular}{|c|c|c|c|c|c|}
\hline Betrieb & Branche & $\begin{array}{l}\text { Beschäf- } \\
\text { tigten- } \\
\text { zahl }\end{array}$ & $\begin{array}{l}\text { Leih- } \\
\text { arbeits- } \\
\text { quote }\end{array}$ & $\begin{array}{l}\text { Zahl der } \\
\text { Interviews }\end{array}$ & $\begin{array}{l}\text { Einordnung in die } \\
\text { Typologie }\end{array}$ \\
\hline 1 & Elektro/nik & 3.000 & $11 \%$ & 1 & Partielle Interessenvertretung \\
\hline 2 & Elektro/nik & 1.741 & $13 \%$ & 1 & Partielle Interessenvertretung \\
\hline 3 & Elektro/nik & 1.500 & $25 \%$ & 1 & Partielle Interessenvertretung \\
\hline 4 & $\begin{array}{l}\text { Schreib-/ } \\
\text { Zeichen- } \\
\text { geräte }\end{array}$ & 979 & $1-2 \%$ & $1+2^{*}$ & Betriebskulturelle Marginalie \\
\hline 5 & Gießerei & 714 & $20 \%$ & $2+4^{*}$ & Integration \\
\hline 6 & Elektro/nik & 581 & $9 \%$ & 1 & $\begin{array}{l}\text { Harmonistische Interessen- } \\
\text { deutung }\end{array}$ \\
\hline 7 & Eletro/nik & 527 & $1-2 \%$ & 1 & Betriebskulturelle Marginalie \\
\hline 8 & Elektro & 333 & unter $1 \%$ & $1+3^{*}$ & Betriebskulturelle Marginalie \\
\hline 9 & Elektro/nik & 307 & $10 \%$ & 1 & $\begin{array}{l}\text { Segmentäre Interessen- } \\
\text { vertretung }\end{array}$ \\
\hline 10 & $\begin{array}{l}\text { Maschi- } \\
\text { nenbau }\end{array}$ & 108 & $11 \%$ & 1 & Partielle Interessenvertretung \\
\hline
\end{tabular}

Bei den mit * bezeichneten Interviews handelte es sich um Gespräche mit Leiharbeiterinnen und Leiharbeitnehmern, bei den übrigen um Gespräche mit Betriebsratsmitgliedern. Neben den in derTabelle aufgeführten betrieblichen Interviews wurde je ein Gespräch mit dem Geschäftsführer eines örtlich tätigen Leiharbeitsunternehmens sowie mit einer Mitarbeiterin/einem Mitarbeiter der örtlichen Arbeitsagentur geführt, die für die Vermittlung von in Leiharbeit Beschäftigten zuständig war.

Quelle: Zusammenstellung der Autorin. diese Beschäftigtengruppe zuständig fühlt, die ja formal nicht Teil der Belegschaft ist, für die er einen Vertretungsauftrag hat. Eine Verantwortungsübernahme auch für die sogenannte Randbelegschaft bedeutet für die Betriebsräte oft erheblichen Mehraufwand, auf den weder ihre personellen Ressourcen noch ihre rechtlichen Mitbestimmungsmöglichkeiten zugeschnitten sind. Trotz der kürzlich leicht verbesserten Gesetzgebung sowie Rechtsprechung bietet das Betriebsverfassungsgesetz kaum rechtliche Eingriffsmöglichkeiten in Sachen Leiharbeit. Dennoch gilt: Der Versuch, Leiharbeitsverhältnisse durch Betriebsratshandeln zu beeinflussen, ist realistischerweise eher in den Entleihbetrieben umzusetzen, insbesondere in solchen, in denen professionelle Mitbestimmungsstrukturen und gewerkschaftliche Vertretungsmacht existieren. Die Kampagne der IG Metall hat gezeigt, dass hier Spielräume existieren, die von Betriebsräten für den Abschluss von Betriebsvereinbarungen (sogenannte Besservereinbarungen) genutzt werden können (vgl. Weinkopf/Vanselow 2008). In der Tarifrunde 2012 (und auch in einigen Unternehmenstarifverträgen) vereinbarte die IG Metall zudem eine tarifliche Stärkung der Mitbestimmungsrechte von Betriebsräten bezüglich der Einsatzbedingungen von Beschäftigten in Leiharbeit. Welche Handlungsmöglichkeiten Betriebsräte bei der Interessenvertretung von Leiharbeitern und Leiharbeiterinnen besitzen und welche Strategien sie konkret verfolgen, ist bislang - sieht man von der Pilotarbeit von Wassermann/Rudolph (2007) einmal $\mathrm{ab}$ - wenig erforscht. Diese Fragen standen daher im Zentrum einer kleineren empirischen Studie, die Leiharbeit als Gegenstand von Aushandlungsprozessen zwischen Geschäftsleitungen, Betriebsräten und Beschäftigten untersuchte. ${ }^{1}$

Zwischen Juni und August 2011 wurden 22 qualitative Interviews in zehn Mittel- und Großbetrieben im Organisationsbereich der IG Metall durchgeführt. Die Studie fand damit nach der „Hochphase“ der Leiharbeitskampagne der IG Metall, aber noch vor den genannten rechtlichen und tariflichen Re-Regulierungsmaßnahmen statt. Auswahlkriterien für die Untersuchungsbetriebe waren die Teilnahmebereitschaft des Betriebsrats und der Versuch, möglichst unterschiedliche Formen des betrieblichen

1 Die Studie wurde im Rahmen eines Lehrforschungsprojekts am Institut für Soziologie der Universität NürnbergErlangen durchgeführt und von der Hans-Böckler-Stiftung finanziell unterstützt. An dem Projekt partizipierten - mit sehr unterschiedlichem Zeit- und Arbeitsaufwand - sieben Studierende, nämlich Monika Gaubitz, Anna Janzen, Thomas Krombach, Felix RoßmeißI, Lisa Schäfer, Johanna Schmidt und Cornelia Stenzel. Judith Holland war als wissenschaftliche Mitarbeiterin beteiligt.

2 Für weitere Angaben zur Methode und zum Untersuchungssample vgl. den Endbericht des Projekts 2011-474-2 (Artus/RoßmeißI 2012). Dieser kann bei der Hans-BöcklerStiftung oder der Autorin angefordert werden. 
Leiharbeitseinsatzes sowie differente Mitbestimmungskulturen einzubeziehen. ${ }^{2}$ Tabelle 1 gibt einen Überblick über wesentliche Merkmale der Untersuchungsbetriebe, die Anzahl der pro Betrieb geführten Interviews sowie die (näherungsweise) Einordnung in die im Folgenden dargestellte Typologie zum Thema „Leiharbeit und Mitbestimmung".

Tabelle 1 zeigt, dass die hier präsentierten Ergebnisse auf einer beschränkten Empirie basieren - sowohl was die „Breite“ als auch die „Tiefe“ des Datenmaterials angeht. ${ }^{3}$ In jedem Untersuchungsbetrieb wurde zwar mindestens ein ausführliches, meist mehrstündiges qualitatives Interview mit einem oder mehreren Mitgliedern des Betriebsrats durchgeführt (in einem besonders interessanten Betrieb, vgl. Abschnitt 3.4, waren es zwei Gespräche); lediglich in drei Untersuchungsbetrieben wurde die Perspektive der Betriebsräte aber durch Gespräche mit Leiharbeiternehmerinnen und Leiharbeitnehmern ergänzt - nämlich in Fällen, in denen Interviews während der Arbeitszeit unproblematisch möglich waren. Die Perspektive des Managements fehlt komplett. Somit können im Folgenden vor allem strategische Überlegungen und Legitimationsweisen der Betriebsräte im Umgang mit Leiharbeit detailliert nachgezeichnet werden. Für eine empirisch belastbare Analyse der konkreten Praktiken des Mitbestimmungshandelns hätte es zusätzlicher Interviews, v.a. mit dem Management bedurft. Der folgende Versuch, eine Typologie von Mitbestimmungshandeln zum Thema Leiharbeit zu konstruieren, ist somit keineswegs empirisch gesättigt. Es handelt sich vielmehr um eine erste, teils noch tentative Verknüpfung empirischer Beobachtungen mit theoretisch-logischen Überlegungen. Diese profitiert von umfangreichen Vorarbeiten der Erlanger Forschungsgruppe Arbeits- und Industriesoziologie zum Thema „betriebliches Interessenhandeln“ (vgl. Bosch et al. 1999; Artus et al. 2001). Insofern handelt es sich auch um den Versuch, eine vor rund 20 Jahren in einem sehr ähnlichen Forschungsterrain erarbeitete Typologie politischer Kulturen innerbetrieblicher Austauschbeziehungen zwischen Betriebsrat und Management fruchtbar zu machen für die Analyse von Mitbestimmungshandeln in Bezug auf Leiharbeit.

Im Folgenden wird zunächst das Konzept der Typenbildung expliziert (Abschnitt 2); anschließend werden verschiedene Varianten von Mitbestimmung zum Thema Leiharbeit dargestellt (3). Neben Betrieben, in denen Leiharbeit aufgrund ihrer quantitativen Beschränktheit mitbestimmungspolitisch unproblematisch ist (3.1), existieren Vertretungsstrategien, in denen die speziellen Interessen von Leiharbeitsbeschäftigten von den Betriebsräten kaum berücksichtigt werden (3.2), in denen die Betriebsräte diese partiell „mitvertreten“ (3.3) oder sie sogar voll in die Vertretungsarbeit integrieren (3.4). Ein Fazit fasst die Ergebnisse in ihrer wissenschaftlichen wie politischen Relevanz zusammen (4).

\section{Typrelevante Dimensionen: Prekari- tätskonstellation und politische Kul- tur der Austauschbeziehungen}

Theoretisch wie empirisch lässt sich die These begründen, dass besonders zwei Faktoren wichtig sind für die Art und Weise, wie Betriebsräte das Thema Leiharbeit in ihre Vertretungspraxis integrieren: erstens, die betriebliche Prekaritätskonstellation, d. h. das Ausmaß, die betriebliche Geschichte sowie Funktion von Leiharbeit, und zweitens, die historisch etablierte politische Kultur innerbetrieblicher Austauschbeziehungen zwischen Betriebsrat und Geschäftsleitung (vgl. Bosch et al. 1999; Artus et al. 2001).

Für eine Typisierung der Prekaritätskonstellation lässt sich die Studie von Holst (2009) nutzen, in der unterschiedliche Formen des Einsatzes von Leiharbeit unterschieden werden: demnach existieren einerseits Strategien, bei denen Leiharbeit eher ausnahmsweise zur Bewältigung von Auftragsspitzen und als kurzfristiger Ersatz bei Absentismus bzw. personellen Engpässen (durch Krankheit, Schwangerschaft etc.) genutzt wird. Diese „situative ad-hoc-Nutzung” (ebd.) fand sich in drei Untersuchungsbetrieben unseres Samples. Die Leiharbeitsquote betrug hier ca. 1-2 \% der Belegschaft (Tabelle 1). In den übrigen sieben Betrieben - darunter auch zwei „Intensivnutzer“ mit einer Leiharbeitsquote von über $20 \%$ - ließ sich hingegen eine strategische Dauernutzung des Instruments der Leiharbeit als zweite Variante finden. Bezogen auf die Frage der Mitbestimmungspolitik war es dabei wenig relevant, ob die in Leiharbeit Beschäftigten primär als „Flexibilitätspuffer“ für Krisenzeiten oder/und für "die dauerhafte Minimierung der Lohnkosten” eingesetzt wurden (Holst 2009). Häufig waren beide Motive relevant und nur schwer voneinander zu trennen. Während die situative ad-hoc-Nutzung - unabhängig von der etablierten betrieblichen Mitbestimmungskultur - zur Folge hatte, dass Leiharbeit eine „betriebskulturelle Marginalie“ darstellt (3.1), sind in Betrieben mit strategischer Dauernutzung von Leiharbeit die Vertretungspolitiken der Betriebsräte in Bezug auf die prekäre „Randbelegschaft“ unterschiedlich (3.2-3.4). Sie differieren - so die These - insbesondere entlang der histo-

3 Die Validität der Daten und Einschätzungen aus den Interviews haben wir allerdings durch wiederholte Diskussionen mit der örtlichen IG Metall-Verwaltungsstelle sowie eine Rückspiegelung der Ergebnisse an die Betriebsräte erhöht. Ein Mitglied der Forschungsgruppe, Felix Roßmeißl, war zudem im Vorfeld der Studie als Praktikant einen Monat lang in der Verwaltungsstelle beschäftigt. Er führte eine Vorstudie durch (vgl. dazu Artus/Roßmeiß। 2012) und erwarb durch zahlreiche Betriebsbesuche umfangreiches betriebsspezifisches Wissen. Ein Teil der Untersuchungsbetriebe war der Erlanger Forschungsgruppe für Arbeits- und Industriesoziologie zudem bereits aus früheren Untersuchungen bekannt. 
risch etablierten Interaktionskultur zwischen Management und Betriebsrat. Damit sind die Routinen, wechselseitig geteilten Normen und Selbstverständlichkeiten der Verhandlungsbeziehungen zwischen den Betriebsparteien gemeint, die das Ergebnis der betrieblichen Interaktionsgeschichte sind. Ohne die Kategorien von Bosch et al. (1999) in ihrer - für die vorliegende eher schmale Empirie deutlich zu differenzierten - Gesamtheit aufzunehmen, ${ }^{4}$ geht die folgende Typologie davon aus, dass die Frage, ob das Verhältnis zwischen Betriebsrat und Geschäftsleitung eher konfliktorisch, kooperativ oder harmonistisch gestaltet ist, wichtig ist für den Umgang der Betriebsräte mit dem Thema Leiharbeit. Dabei wird kein deterministisches Eins-zueins-Entsprechungsverhältnis der jeweils etablierten Interaktionskultur und der Einbeziehung prekär Beschäftigter in die Interessenvertretung behauptet. Die jeweiligen Mitbestimmungskulturen bilden jedoch einen Möglichkeitsraum, innerhalb dessen die Wahl bestimmter Vertretungsstrategien mehr oder weniger wahrscheinlich ist. Die Spaltung in eine „Kern-“ und eine „Randbelegschaft" hat nämlich Folgen für die zur Verfügung stehenden mitbestimmungspolitischen Machtressourcen. Dies gilt insbesondere für die Möglichkeiten der Belegschaftsmobilisierung, die für interessenbetonte und konfliktorische Interaktionsmodi eine unabdingbare Voraussetzung, eine „conditio sine qua non“ darstellt. Die Integration auch prekärer Belegschaftsgruppen in die Vertretungsstrategien des Betriebsrats erscheint daher dringend geboten (3.4.). Kooperativ agierende Betriebsräte haben im Regelfall im betrieblichen Alltag nur einen eingeschränkten direkten Bezug zur Belegschaft. Sie nutzen primär rechtliche Machtmittel, häufig in strategischer Weise. Angesichts der stark beschränkten rechtlichen Mitbestimmungsmöglichkeiten zum Thema Leiharbeit ist die Einbeziehung der prekären Randbelegschaft in diese Strategien - trotz des häufig anzutreffenden „politischen Willens“ - äußerst mühsam und bleibt im Regelfall partiell (3.3). Stärker harmonistisch angelegte Betriebsratsstrategien, die gemeinsame Interessen von Kapital und Arbeit betonen, werden durch den Einsatz von Leiharbeitern hingegen kaum unmittelbar tangiert. Die weitgehende Untätigkeit dieser Betriebsräte in Sachen Leiharbeit kann dabei unterschiedlich legitimiert werden (3.2).

Im Folgenden werden verschiedene Mitbestimmungskulturen zum Thema Leiharbeit beschrieben, die entlang der o.g. Dimensionen variieren. Entscheidend ist zunächst, ob Leiharbeit im Unternehmen eine betriebskulturelle Marginalie darstellt (3.1) oder ein quantitativ relevantes sowie dauerhaftes Phänomen ist, das eine Aufspaltung der Belegschaft in einen „Kern“ und einen „Rand“ zur Folge hat und damit eine betriebsinterne Konkurrenzsituation konstituiert. Nur im letzteren Fall existiert für den Betriebsrat ein virulenter Problemdruck und er ist gezwungen, sich aktiv zum Thema Leiharbeit zu verhalten - sei es, indem er einen besonderen Vertretungsauftrag für die Leiharbeitnehmer im Betrieb als unnötig negiert oder explizit ablehnt
(3.2), dass er deren Interessen mit reduziertem Engagement neben der Stammbelegschaft quasi mitvertritt (3.3), oder indem er versucht, prekär Beschäftigte in gleichem Maße wie Stammbeschäftigte zu vertreten (3.4). ${ }^{5}$

\subsection{Leiharbeit als betriebskulturelle Marginalie}

In Betrieben mit einem geringen Umfang von Leiharbeit (ca.1-2 \%) ist das Thema innerbetrieblich kaum politisiert - weder im Verhältnis zwischen Betriebsrat und Management noch zwischen Betriebsrat und Belegschaft oder zwischen Stamm- und Randbelegschaft. Dies gilt unabhängig von der jeweils etablierten Mitbestimmungskultur. Der Betriebsrat hat nur geringe Legitimationsnotwendigkeiten für seine Politik zum Thema Leiharbeit. Am ehesten existieren diese noch in seinem Verhältnis zur IG MetallVerwaltungsstelle. Die Regelungsintensität sowie Normierungssituation kann daher stark variieren. Wenn sich der Betriebsrat jedoch für die Frage der betrieblichen Regelung von Leiharbeit einsetzt, so gestaltet sich z. B. der Abschluss einer Betriebsvereinbarung meist unproblematisch. Sie betrifft ja nur einen sehr geringen Teil der Belegschaft, tangiert keinen strategisch wichtigen Bereich der Personalpolitik und stellt auch kaum einen relevanten Kostenfaktor dar. Das Verhältnis zwischen Stamm- und Randbelegschaft ist häufig ausgesprochen gut und spannungsfrei - gerade weil in Leiharbeit Beschäftigte ganz klar eine Sonderrolle im Unternehmen spielen. Ihr Einsatz ist zeitlich strikt befristet und es existieren nur beschränkte Übernahmemöglichkeiten. Die Grenzen zwischen „Kern“ und „Rand“ sind damit klar konturiert und es existiert kaum eine direkte Konkurrenzsituation. Gerade wegen der klaren Ausgrenzung der Leiharbeiter sind die sozialen Beziehungen zwischen Stamm- und Randbelegschaft im Regelfall entspannt, aber auch von deutlicher Hierarchie geprägt. Und: Sogar in Betrieben, in denen Leiharbeit eine Randerscheinung ist, äußern die Betriebsräte die Einschätzung, dass die Existenz von Leiharbeit gewisse disziplinierende Wirkungen auf die Stammbelegschaft habe.

Summa summarum ist die Repräsentationssituation in diesen Betrieben meist davon geprägt, dass auch die Interessen der Leiharbeitnehmerinnen und Leiharbeitnehmer im Alltagsgeschäft „mitvertreten“ werden. Dies ist - angesichts ihrer geringen Anzahl - weder besonders problematisch noch besonders zeitaufwendig. Und doch hat

4 Bosch et al. (1999) haben sechs verschiedene Interaktionsmuster identifiziert, die vor allem nach der Interessendefinition variieren, d. h. ob eher divergierende oder gemeinsame Interessen von Kapital und Arbeit betont werden.

5 Theoretisch wie praktisch existiert zudem die Möglichkeit einer Mitbestimmungspolitik, die auf die komplette Vermeidung von Leiharbeit zielt. Dies kam im Untersuchungssample nicht vor. Auf die diesbezügliche Ergänzungsbedürftigkeit der hier vorgestellten Typologie sei jedoch hingewiesen. 
die Integration der als Leiharbeitnehmer Beschäftigten in die Belegschaft ihre deutlichen Grenzen, die eingehalten werden müssen, wenn ihre Existenz kulturell unproblematisch bleiben soll.

\subsection{Harmonistische oder segmentäre Interes- senvertretung}

Alle im Folgenden diskutierten Mitbestimmungskulturen zum Thema Leiharbeit sind dadurch gekennzeichnet, dass Leiharbeit innerbetrieblich ein quantitativ relevantes Phänomen ist (d. h. die Leiharbeitsquote liegt über $10 \%$ ). Dies führt im Regelfall zu virulenten Interessengegensätzen zwischen Betriebsrat und Geschäftsleitung, zwischen Stamm- und Randbelegschaft, zum Teil auch zwischen Betriebsrat und Belegschaft. Der Betriebsrat muss sich auch bezüglich der Frage positionieren, welche Relevanz er betrieb(swirtschaft)lichen Interessen einräumt und welche Rolle überbetriebliche, gewerkschaftliche Solidaranforderungen für ihn spielen. Nur in einem einzigen Fall negierte ein Betriebsrat die schiere Problematik dieser Situation. Er argumentierte in einer Weise, die sich als harmonistische Interessendeutung bezeichnen lässt, $\mathrm{d}$. h. er ging von einer weitgehenden Interessenidentität von Geschäftsleitung, Betriebsrat, Stammbelegschaft und Randbelegschaft aus. Im Grunde sei der Einsatz der Leiharbeiter doch vorteilhaft für alle, denn, so das Zitat, „sie geben dem Unternehmen eine gewisse Sicherheit in der längerfristigen Planung", was sowohl der Geschäftsleitung als auch der Stammbelegschaft nütze. Und auch die Leiharbeitnehmer bekämen dadurch „eine Chance, einen Fuß in die Tür zu bringen” (Interview). Hintergrund dieser harmonistischen Interessendeutung waren im konkreten Fall drei recht spezielle Rahmenbedingungen: Erstens war die Leiharbeitsquote von knapp $10 \%$ ein noch recht junges Phänomen, das erst nach der Wirtschaftskrise 2008/09 Einzug gehalten hatte. Zweitens existierte bislang eine Übernahmepraxis, die vom konjunkturellen Boom des Unternehmens geprägt war. Der Betrieb übernahm zuvor in Leiharbeit Beschäftigte ab einer Betriebszugehörigkeit von sechs bis zwölf Monaten zuverlässig in ein befristetes Beschäftigungsverhältnis. Ein ,informelles Gentleman-Agreement" (Betriebsratszitat) zwischen Betriebsrat und Geschäftsleitung hatte bislang ausgereicht, um dieses - für die meisten Leiharbeitnehmer primäre Vertretungsinteresse zu befriedigen. Drittens existierte in dem Betrieb eine stark integrationsorientierte und harmonisch angelegte Interessenvertretungskultur, in der z. B. ein Rückgriff des Betriebsrats auf die Mobilisierung der Belegschaft als Machtressource nahezu ausgeschlossen schien. Der Betriebsrat betrieb eine ausgeprägte Stellvertreterpolitik, was mit einem recht geringen Informationsstand zum Thema Leiharbeit gepaart war. So konnte er im Interview nicht einmal angeben, was im Betrieb beschäftigte Leiharbeiter verdienen. Theoretisch fühlte er sich zwar für diese Beschäftigtengruppe ebenso zuständig wie für die Stammbelegschaft („Ich finde, das [die Vertretung der Leiharbeiter] gehört zu unserem Job“ - Interview). Praktisch schien er jedoch ein eher distanziertes Verhältnis zur Belegschaft insgesamt - also nicht nur zu den Leiharbeitnehmern - zu haben. Gerade die offenbar recht große Distanz zwischen Belegschaft und Betriebsrat erleichterte es ihm möglicherweise, sich zumindest akklamatorisch gleichermaßen für alle zuständig zu fühlen und Interessenkonflikte zwischen den von ihm repräsentierten Beschäftigten nicht allzu deutlich wahrzunehmen.

$\mathrm{Ob}$ eine solche harmonistische Interpretation der betrieblichen Interessenlagen jedoch auf Dauer gelingen kann in einem Unternehmen, das in größerem Umfang und kontinuierlich Leiharbeit einsetzt, scheint eher fraglich. Wahrscheinlicher ist, dass Betriebsräte, die eine integrationsorientierte oder harmonistische Verhandlungskultur mit dem Management pflegen, diese Kultur wahren, indem sie eine explizit segmentäre Interessenvertretungspolitik betreiben. In diesen Fällen werden zwar virulente Interessenunterschiede und auch -gegensätze zwischen Stamm- und Randbelegschaft wahrgenommen. Der Betriebsrat entscheidet sich jedoch klar und ausschließlich für die Repräsentation der Festangestellten. Anders als im Fall harmonistischer Interessendeutung mag Leiharbeit dann vom Betriebsrat als Beschäftigungsform explizit abgelehnt und sogar skandalisiert werden. Diese Einschätzung bleibt jedoch folgenlos für seine Betriebsratspraxis. Das potenziell konflikthafte Thema der Leiharbeit wird - ebenso wie im oberen Fall aus dem harmonistischen Verhältnis zur Geschäftsleitung ausgeklammert, jedoch nicht, indem widersprüchliche Interessenlagen von Leiharbeitnehmern und Stammbelegschaft geleugnet, sondern indem die Leiharbeiter als Klientel der Repräsentationsarbeit vom Betriebrat schlicht negiert werden. Der Betriebsrat affirmiert seine eng gesetzten rechtlichen Befugnisse. Er betont, dass er rechtlich weder ein Mandat noch ausreichende Machtbefugnisse besitze, um sich um die Belange der als Leiharbeitnehmer Beschäftigten zu kümmern. Die Klage über deren „Ausbeutung“ ist typischerweise gepaart mit der Betonung der eigenen Machtlosigkeit und Nicht-Zuständigkeit: „Ich kenn welche [Leiharbeiter], die arbeiten mit $200 \%$ jeden Tag. Ich frag' mich, wie lang halten die das durch, weil irgendwann verschleißen sie. Verschleißen nur, um den täglichen Broterwerb zu sichern, der noch nicht mal ausreicht. Und da tun sie mir eigentlich leid. Kann aber nix ändern“" (Interview). Dennoch kommt es freilich vor, dass sich Leiharbeiternehmer an den Betriebsrat wenden und er kümmert sich zuweilen auch um ihre Anliegen. Dies interpretiert er jedoch eher als Übererfüllung seiner Aufgaben, ja sogar als illegitime Überschreitung seiner Befugnisse. Die moralische Empörung über prekäre Arbeitsbedingungen und auch das Mitleid mit den Betroffenen bleiben folgenlos für seine Politik, denn die Verantwortung wird ausschließlich betriebsexternen Instanzen zugeschrieben: den profitgierigen Leiharbeitsfirmen, dem zynischen Gesetzgeber, den unfähigen Gewerkschaften. Auch den Leiharbeitern selbst wird ein gewisses $\mathrm{Maß}$ an Mitschuld am eigenen Schicksal gegeben. 
Geschäftsleitung und Betriebsrat hingegen scheinen weitgehend unbeteiligt an den „schlechten Verhältnissen“.

Harmonistische Interessendeutungen wie segmentäre Vertretungsstrategien perpetuieren somit den „betrieblichen Effizienzpakt" zwischen Betriebsrat und Geschäftsleitung, indem sie die Bedeutung spezifischer Interessen von prekär Beschäftigten wie auch gewerkschaftliche Solidaransprüche negieren. Typisch ist, dass keine Betriebsvereinbarung zum Thema Leiharbeit existiert und diese auch nicht angestrebt wird. Das Niveau der Entlohnung und die Übernahmepraxis sind dementsprechend schlecht. Eine gewisse Regelungsroutine gibt es allenfalls über informelle Absprachen. Während der Betriebsrat im Rahmen harmonistischer Interessendeutung einen besonderen Vertretungsauftrag für Leiharbeiternehmerinnen und Leiharbeitnehmer leugnet, indem er eine Differenzierung zwischen Stamm- und Randbelegschaft tendenziell zu verwischen sucht, konstruieren und verstärken segmentär agierende Betriebsräte aktiv die Unterscheidung zwischen Stamm- und Randbelegschaft. Sie pochen darauf, dass als Leiharbeitnehmer Beschäftigte nicht zur Belegschaft des Einsatzbetriebs gehören. Die Legitimität und sogar Unausweichlichkeit einer Mitbestimmungskultur "jenseits der Leiharbeitnehmer“ wird dabei aus den gesetzlichen Vorgaben abgeleitet. „Da sind schon einige [Leiharbeiter] da gewesen und sagen: ,Warum kann die Firma nichts drauf bezahlen?' Da sag ich: ,Da sind Sie, wenn wir was draufbezahlen, Mitarbeiter von uns. Das sind Sie nicht. Und dann dürfen wir nicht. Gehen Sie zu Ihrer [Leiharbeits] Firma und sagen: 'Wir wollen mehr Geld haben' [fiktive Antwort des Gegenübers:] ,Nee, die geben nichts. Dann sag ich: ,Na, ich kann Ihnen auch nichts geben“" (Interview).

\subsection{Partielle Interessenvertretung für Leihar- beitsbeschäftigte: permanentes Tauziehen}

In unserer Studie, die sich im Kern des Organisationsbereichs der IG Metall bewegte, war die völlige Negierung eines Repräsentationsauftrags der Betriebsräte für die Leiharbeitnehmerinnen und Leiharbeitnehmer die Ausnahme. Die Regel war der Anspruch und auch die Praxis, diese Beschäftigtengruppe partiell mitzuvertreten. Dieser Anspruch leitete sich sowohl aus gewerkschaftlichen Überzeugungen wie auch aus dem Bewusstsein machtpolitischer innerbetrieblicher Vertretungsnotwendigkeiten ab. In den einschlägigen Betrieben herrschen i. d. R. kooperativ angelegte Interaktionsmodi, bei denen differente Interessen von Belegschaft und Geschäftsleitung anerkannt werden und versucht wird, diese kompromisshaft zu vermitteln. Solche professionellen, weitgehend sachlichen und auf gegenseitigen Austausch orientierten „do-ut-des“-Beziehungen sind zwar nicht typisch für die Gesamtheit des Mitbestimmungshandelns in Deutschland (vgl. Artus 2013); in Großbetrieben der westdeutschen Metall- und Elektroindustrie sind sie jedoch weit verbreitet. Sie beruhen primär auf einer Kultur rationaler Argumentation, implizieren aber zugleich den
Einsatz einer breiten Palette von Machtmitteln. Für die Betriebsräte ist die strategische Nutzung des Betriebsverfassungsgesetzes zentral, aber auch die Mobilisierbarkeit der Belegschaft ist ein meist latentes, dennoch wichtiges Drohpotenzial, das (nur) im Ausnahmefall auch genutzt wird (vgl. Bosch et al. 1999). Die Spaltung der Beschäftigten in Stamm- und Randbelegschaft tangiert insofern direkt die innerbetrieblichen Machtverhältnisse - und dessen sind sich die Betriebsräte auch bewusst. Problematisch ist, dass sie mit ihrem üblichen Instrumentarium des Betriebsverfassungsgesetzes in der Frage der Leiharbeit nur wenig erreichen können. „Das heißt nichts anderes, als dass wir nun einen politischen Kampf führen hier. Wir führen eine politische Auseinandersetzung, keine rechtliche. Rechtlich werden wir diese Auseinandersetzung verlieren, das ist uns auch klar“"(Interview). Gespräche mit der Geschäftsleitung werden ebenso wie Betriebsversammlungen als Orte der Politisierung genutzt; man droht damit oder nutzt das Recht auf (Nicht)Zustimmung zu Einstellungen als Mittel, um die Nutzung von Leiharbeit zumindest umständlich zu gestalten. „Wir haben halt einfach so ein bisschen Eskalation betrieben, dass die Firma gemerkt hat, wir lassen da nicht locker, wir werden das auch immer wieder ansprechen" (Interview)

Letztendlich nutzen die Betriebsräte die Gesamtheit ihrer betrieblichen Machtmittel, um - im Sinne eines Verhandlungsbudgets - auch das Thema Leiharbeit wenigstens partiell mitzuverhandeln. Dies ist zwar ein steiniger und anstrengender Weg, ein „Kampf gegen Windmühlen“ (Betriebsratszitat); im Ergebnis war die Normierung der Leiharbeit jedoch überall besser als die gesetzlichen und tariflichen Vorgaben. In Betriebsvereinbarungen wurden maximale Obergrenzen des quantitativen Einsatzes von Leiharbeit vereinbart; es wurden zeitliche Übernahmeregeln fixiert, und in allen Fällen wurde die Lohnhöhe gegenüber den tariflichen Vorgaben aufgestockt. Trotz dieser Erfolge blieb die Situation für die Betriebsräte jedoch schwierig: „Das geht an die Substanz. Es ist gar nicht einfach, wenn du jahrelang diese Auseinandersetzung führst“ (Interview). Zugleich sahen sich die Betriebsräte in ihren Bemühungen nur verhalten von der Belegschaft unterstützt. Der Stammbelegschaft wurde eine zumindest ambivalente Interessenposition unterstellt, denn „solange wir Leiharbeitnehmer haben, sind die Stammmitarbeiter ja geschützt" (Interview). Diesen Effekt hatte die erst kürzlich erlebte Wirtschaftskrise nur allzu deutlich vorgeführt. Die Leiharbeitnehmer wiederum können sich meist nicht mit dem vom Betriebsrat verfolgten Ziel einer Beschränkung von Leiharbeit im Unternehmen identifizieren - zumal dann nicht, wenn Löhne und Arbeitsbedingungen auch noch attraktiver gestaltet sind als in den meisten anderen Einsatzbetrieben. Frustrierend ist für viele Betriebsräte auch die Erfahrung, dass Leiharbeiter sich kaum aktiv in innerbetriebliche Politikprozesse einbringen oder manchmal im Zuge von Betriebsratswahlen nicht einmal ihr Wahlrecht in Anspruch nehmen. 
Insgesamt betreiben die Betriebsräte also eine Art „permanentes Tauziehen“ (Betriebsratszitat). Sie integrieren die Leiharbeiter zumindest partiell in ihre Repräsentationsarbeit und versuchen, betriebliche Verwertungsinteressen und betriebsübergreifende Solidarmotive zu vermitteln. Die Trennung zwischen Stamm- und Randbelegschaft bleibt jedoch akzentuiert und von einem aktiven Repräsentationsverhältnis zwischen Betriebsrat und in Leiharbeit Beschäftigten kann kaum die Rede sein. Es handelt sich um eine Interessenvertretung partiell auch für, aber nicht mit den Leiharbeitnehmern.

\subsection{Integration der Leiharbeitsbeschäftigten}

Eine (nahezu) egalitäre Integration von Leiharbeitnehmerinnen und Leiharbeitnehmern in die betriebliche Mitbestimmungspraxis kommt sicherlich nur in Ausnahmefällen vor, im Rahmen unserer Studie nur in einem einzigen Betrieb. ${ }^{6}$ In diesem waren recht spezielle Voraussetzungen gegeben: Der Betriebsrat hatte eine ausgeprägte innerbetriebliche Machtstellung inne, die sowohl auf einer professionellen und in hohem Maße sachkompetenten Betriebsratspolitik beruhte als auch auf einem ausgesprochen engen Verhältnis zwischen Betriebsrat und Belegschaft. Die Beschäftigten waren im Rahmen häufiger betrieblicher Konflikte zuverlässig mobilisierbar und somit gab es ein virulentes Drohpotenzial gegenüber der Geschäftsleitung. Eine weitere Voraussetzung bestand darin, dass der Betriebsrat vor Ort einen direkten Ansprechpartner und "Gegenspieler" in der Geschäftsleitung hatte, der befugt war, betriebliche Entscheidungen zu treffen. Und schließlich zeichnete sich die Konkurrenzsituation des Betriebs dadurch aus, dass das Unternehmen produktionstechnisch gewisse Alleinstellungsmerkmale aufwies, wodurch Existenz und Profitabilität des Betriebs vergleichsweise solide abgesichert waren. Vor diesem Hintergrund war es dem Betriebsrat in einer akuten Krisensituation gelungen, die dringend benötigte Kooperationswilligkeit der Belegschaft von einer Betriebsvereinbarung abhängig zu machen, die ihm faktisch eine Ausweitung seiner Mitbestimmungsrechte garantierte. Einstellungen (auch von Leiharbeitnehmern) sind seitdem nur noch mit Zustimmung des Betriebsrats möglich und diese Zustimmung kann auch nicht durch das Arbeitsgericht ersetzt werden. Zudem wurde eine erhebliche Erhöhung der Stundenlöhne für die (vergleichsweise große Zahl) von (ausschließlich männlichen) Leiharbeitern vereinbart. „Das heißt im Prinzip die Leiharbeiter, wenn sie bei [Betrieb 5] arbeiten, haben sie $1000 €$ brutto mehr. Und das führt mittlerweile dazu, dass Beschäftigte in der Metallindustrie kündigen, weil die nicht tarifgebunden sind und als Leiharbeiter hierherkommen. [...] Also das war, das war natürlich alles konfliktorisch“ (lacht) (Interview).

Die vereinbarte Lohnhöhe für Leiharbeit ist mit Abstand die beste „Besser-Vereinbarung“ in den von uns untersuchten Betrieben. Bei der grundsätzlichen Verurteilung von Leiharbeit als Beschäftigungsform zeigt sich der Betriebsrat hingegen wesentlich zurückhaltender als die meisten seiner Kollegen. Er setzt nicht auf einen fundamentalen Kampf gegen Leiharbeit oder deren quantitative Beschränkung (z. B. über eine Maximalquote), sondern auf die Entprekarisierung und möglichst weitgehende Gleichstellung der Leiharbeiter mit den Stammbeschäftigten.

Der Anspruch auf Egalisierung (statt Vermeidung) von Leiharbeit impliziert, dass der Betriebsrat seinen Vertretungsanspruch nahezu eins zu eins auf die Randbelegschaft ausdehnt. Diese wird wenigstens näherungsweise zur gleichberechtigten Vertretungsklientel. Er nutzt seine betriebliche Machtposition gegenüber der Geschäftsleitung, um Druck auszuüben auf die (einzige) Leiharbeitsfirma, bei der alle im Betrieb eingesetzten Leiharbeiter beschäftigt sind. So gelingt es ihm auch, deren Beschäftigungsbedingungen zu kontrollieren und eine sehr wirksame Schutzfunktion auszuüben: „Wir haben ne Betriebsvereinbarung gemacht, wo wir sozusagen zu unserer Betriebsvereinbarung den Arbeitsvertrag zwischen [der Leiharbeitsfirma] und [Betrieb 5] als Bestandteil nehmen, und wenn die [Leiharbeitsfirma] sich nicht dran halten, dann fliegen die raus und das wissen die auch. Ich hab’ den Geschäftsführer hier gehabt von [der Leiharbeitsfirma] und das wissen die auch, dass wir keinen Spaß machen. Die haben jetzt zweimal, zwei Kollegen [gemeint sind Leiharbeiter] mal gekündigt, die mussten die Kündigungen dann zurücknehmen, obwohl ich da rechtlich also keine Befugnis habe, ja, die wurden gezwungen, die zurückzunehmen“ (Interview).

Insgesamt kann man also davon sprechen, dass der Betriebsrat die Leiharbeiter in nahezu ähnlich effektiver Weise vertritt wie die Stammbeschäftigten. Dies tut er jedoch nicht „umsonst“. Angesichts seiner häufig konflikthaften Vertretungsstrategien, bei denen er auf die Mobilisierbarkeit der Belegschaft angewiesen ist, ist der Betriebsrat besonders sensibel und auch geübt in Fragen der Organisierung der betrieblichen Basis. Allen neu beschäftigten Leiharbeitern wird in einem persönlichen Gespräch deutlich gemacht, dass diese Errungenschaften für Leiharbeiter nicht vom Himmel gefallen sind, sondern auf der aktiven gewerkschaftlichen Organisierung und Mobilisierungsbereitschaft der Beschäftigten beruhen. Der gewerkschaftliche Organisationsgrad der Leiharbeiter liegt unter diesen Umständen bei $100 \%$. Die Betriebsratspolitik zielt also tendenzi-

6 Es wird jedoch die These vertreten, dass die "Integration von Leiharbeitnehmern" in die Mitbestimmungspraxis kein abweichender Einzelfall, sondern eine typische Ausprägung von Mitbestimmungshandeln ist - auch wenn sie möglicherweise empirisch ähnlich selten vorkommt wie konfliktorische Austauschbeziehungen zwischen Betriebsrat und Geschäftsleitung (Bosch et al. 1999). Ein weiterer, ähnlich gelagerter Fall der betrieblichen Durchsetzung erweiterter Mitbestimmungsrechte zum Thema Leiharbeit wurde z. B. auch im Rahmen einer Betriebsfallstudie dokumentiert, die im Anschluss an das hier dargestellte studentische Projekt als Qualifikationsarbeit entstand (vgl. Stenzel 2012). 
ell darauf, die Leiharbeiter als "ganz normale Kollegen“ in den Betrieb und auch in die betriebliche Gewerkschaftskultur zu integrieren - mit (möglichst) gleichen Rechten (in punkto Lohn und sozialer Absicherung) und gleichen Pflichten (in punkto Gewerkschaftsmitgliedschaft und Mobilisierungsbereitschaft).

\section{Resümee}

Die vorab dargestellten Mitbestimmungsvarianten zum Thema Leiharbeit basieren auf einer beschränkten empirischen Basis. Sie geben lediglich einen ersten Einblick, keinesfalls einen erschöpfenden Überblick über mögliche Mitbestimmungsstrategien zum Thema Leiharbeit. Der theorie- wie auch empiriegestützte erste Versuch einer Typenbildung, der insbesondere das Konzept des „betrieblichen Interessenhandelns" (Bosch et al. 1999) als heuristischen Rahmen nutzte, mag aber dennoch helfen, Begriffe sowie Kategorien für weitere Untersuchungen $\mathrm{zu}$ schärfen. Die Studie macht jedenfalls deutlich, dass trotz identischer rechtlicher Vorgaben eine große Heterogenität möglicher Betriebsratspolitiken existiert: Jenseits der eher unproblematischen und wenig politisierten Fälle, in denen Leiharbeit ein betriebliches Schattendasein führt, finden sich vergleichsweise defizitäre Vertretungsstrategien von Betriebsräten - defizitär in dem Sinne, dass die besonderen Interessen der Leiharbeitnehmerinnen und Leiharbeitnehmer entweder nicht gesehen oder bewusst aus der eigenen Repräsentationsarbeit ausgegrenzt werden. Infolgedessen existieren allenfalls informelle Absprachen mit der Geschäftsleitung zum Thema Leiharbeit, und die Arbeitsbedingungen der Randbelegschaft bewegen sich kaum über dem rechtlichen bzw. tariflichen Mindestniveau. In größeren, gewerkschaftlich zufriedenstellend organisierten Metallbetrieben kann eine „partielle Mitvertretung“ der als Leiharbeitnehmer Beschäftigten durch die Betriebsräte als interessenpolitische Normalität gelten. Die Betriebsräte führen hier einen schwierigen betriebspolitischen Kampf für eine Beschränkung der Leiharbeit, für Übernahmen sowie für eine wenigstens stückweise Annäherung an das Ziel „equal pay“. Für diesen Kampf sind sie machtpolitisch und vor allem rechtlich ausgesprochen schlecht ausgestattet. Das „permanente Tauziehen“ ist zwar im Regelfall von partiellen Erfolgen in der Form von sogenannten BesserBetriebsvereinbarungen gekrönt; zugleich zermürbt es jedoch auf Dauer so manchen motivierten Betriebsrat, dessen Bemühungen sowohl von der Stamm- als auch von der Randbelegschaft nur bedingt unterstützt und gewürdigt werden. Nur in Ausnahmefällen gelingt es, eine Betriebsratspolitik durchzusetzen, die Leiharbeiter weitgehend egalitär integriert. Dazu bedarf es einer ausgesprochen starken innerbetrieblichen Machtposition des Betriebsrats, die sich in einer Ausweitung seiner Mitbestimmungsrech- te auch auf die Belange von Leiharbeitsbeschäftigten dokumentiert und sich zudem in der Integration der prekär Beschäftigten in die gewerkschaftliche Solidargemeinschaft ausdrückt.

Sicher ist jedoch: Obwohl die rechtlichen Eingriffsmöglichkeiten von Betriebsräten beim Thema Leiharbeit gering sind, haben sie in der Praxis vielfach einen großen Einfluss auf die Normierung von Arbeits- und Entlohnungsbedingungen. Mitbestimmung macht einen Unterschied - oder auch: Strategy matters. Die Frage, ob Betriebsräte sich zuständig fühlen für die Vertretung der Randbelegschaft und einen Teil ihrer Macht-, Zeit- und Einflusspotenziale für dieses Ziel nutzen, hat direkte Folgen für die Beschäftigungsbedingungen des betrieblichen Prekariats, das rechtlich gesehen nur bedingt in ihren mitbestimmungspolitischen Zuständigkeitsbereich fällt, faktisch jedoch vor Ort im Betrieb arbeitet und allein durch seine Präsenz eine moralische Herausforderung an gewerkschaftliche Solidaritätsnormen darstellt.

Wie erwähnt, wurde die Studie zu einem Zeitpunkt durchgeführt, zu dem der allgemeinverbindliche Mindestlohn in der Zeitarbeit, die neuen Bestimmungen des Arbeitnehmerüberlassungsgesetzes, die Branchenzuschläge im Bereich der IG Metall sowie die tariflich erweiterten Vertretungsmöglichkeiten der Betriebsräte noch nicht in Kraft waren. Wichtige Analyseperspektiven für zukünftige Studien wären daher die Folgen dieser Re-Regulierungsmaßnahmen für die Betriebsratspraktiken und die Normierungssituation im Bereich der Leiharbeit: Werden die neuen Regeln eingehalten bzw. genutzt? Gestalten sich die Arbeits- und Entlohnungsbedingungen der Leiharbeitnehmer dadurch nicht nur besser, sondern auch einheitlicher? Fühlen sich die segmentär agierenden Interessenvertreter angesichts der neuen Rechte nun verantwortlicher für die Randbelegschaften? Bewirken die neuen Mindestnormen einen „Sog nach oben“ auch in jenen Betrieben, in denen die (nunmehr) tariflichen Vorgaben längst im Rahmen von Betriebsvereinbarungen Gültigkeit besaßen? Wie verhalten sich kollektive Normierungen und betriebliche Praktiken zueinander?

Zudem ist zu betonen, dass die dargestellten typischen Mitbestimmungsvarianten die Verhältnisse in Kernbereichen gewerkschaftlicher Kampfkraft abbilden. Die Leiharbeits-Kampagne der IG Metall hat hier in der Vergangenheit Grundlagenarbeit für eine Politisierung des Themas geleistet. Die Machtverhältnisse zwischen Betriebsrat und Geschäftsleitung dürften zudem bezogen auf den bundesdeutschen Durchschnitt deutlich positiver gestaltet sein als dies in kleineren Unternehmen, in vielen Dienstleistungsbranchen oder in Ostdeutschland der Fall ist. Leiharbeit, die etwa in Call-Centern, in der mobilen Pflege oder in Schlachthöfen - weit jenseits gewerkschaftlicher Kernzonen - stattfindet, profitiert nicht nur in geringerem Maße von den o.g. kollektiven Re-Regulierungen, sie hat bisher auch nur wenig wissenschaftliche Aufmerksamkeit auf sich gezogen. Dabei belegen einschlägige Statistiken seit Jahren eine zunehmen- 
de Tertiarisierung von Leiharbeit. Hier ist in Zukunft noch wichtige wissenschaftliche und auch interessenpolitische Grundlagenarbeit zu leisten.

Die obige Mitbestimmungstypologie gibt insofern Einblick in vergleichsweise privilegierte Zonen der Interessenvertretung und der Leiharbeit - und selbst hier gilt: Die demokratischen Vertretungs- und Partizipationschancen von Leiharbeitsbeschäftigten sind deutlich prekär, d. h. negativ abweichend vom gesellschaftlichen Durchschnitt. Jenseits der mitbestimmungspolitischen Kernbereiche dürften segmentäre Vertretungsstrategien oder harmonistische Situationsdeutungen von Betriebsräten noch wesentlich häufiger auftreten - sofern es überhaupt Betriebsräte gibt. Alles in allem müssen die Ergebnisse der Studie daher ausgesprochen bedenklich stimmen in Bezug auf die Möglichkeiten innerbetrieblicher demokratischer Teilhabe prekär Beschäftigter. Eine rechtliche oder auch tarifliche Stärkung der Betriebsräte beim Thema Leiharbeit scheint dringend geboten. Der jüngste Tarifabschluss der IG Metall ist daher sicherlich ein Schritt in die richtige Richtung - wenngleich angesichts der Größe des Problems eher ein Trippelschritt. Mehr rechtliche Möglichkeiten der Betriebsräte beim Thema Leiharbeit könnten die deregulierende Wirkung dieser Beschäftigungsform beschränken. Eine verbesserte Einbeziehung der Randbelegschaften in die Mitbestimmungspraxis der Betriebsräte könnte den aktuell beobachtbaren Prozess der Entdemokratisierung der Erwerbsarbeitssphäre verlangsamen. Grundlegende Erfolge gegen die Prekarisierung der Arbeitswelt bedürfen allerdings vermutlich des Überdenkens der 2004 erfolgten gesetzlichen Liberalisierungen.

\section{LITERATUR}

Artus, I. (2012): Weiterentwicklung nicht versäumen, in: Mitbestimmung (6), S. $37-38$

Artus, I. (2013): Precarious delegates: irregular forms of employee interest representation in Germany, in: Industrial Relations Journal, 44 (4), S. 409-424 Artus, I./Liebold, R./Lohr, K./Schmidt, E./Schmidt, R./Strohwald, U. (2001): Betriebliches Interessenhandeln. Band 2: Zur politischen Kultur der Austauschbeziehungen zwischen Management und Betriebsrat in der ostdeutschen Industrie, Opladen

Artus, I./RoßmeißI, F. (2012): Leiharbeit und prekäre Arbeit - aktuelle Situation und Trends im Organisationsbereich der bayerischen IG Metall, interner Endbericht an die Hans-Böckler-Stiftung (Projekt-Nr. 2011-474-2), Erlangen, Oktober 2012 Bellmann, L./Kühl, A. (2007): Weitere Expansion der Leiharbeit? Eine Bestandsaufnahme auf der Basis des IAB-Betriebspanels, Abschlussbericht an die Hans-Böckler-Stiftung, Düsseldorf

Bosch, A./Ellguth, P./Schmidt, R./Trinczek, R. (1999): Betriebliches Interessenhandeln. Band 1: Zur politischen Kultur der Austauschbeziehungen zwischen Management und Betriebsrat in der westdeutschen Industrie, Opladen Brehmer, W./Seifert, H. (2007): Wie prekär sind atypische Beschäftigungsverhältnisse? Eine empirische Analyse, WSI-Diskussionspapier (156), Düsseldorf, http://www.böckler.de/pdf/p_wsi_diskp_156.pdf

Brinkmann, U./Dörre, K./Röbenack, S. (2006): Prekäre Arbeit. Ursachen, Ausmaß und subjektive Verarbeitungsformen unsicherer Beschäftigungsverhältnisse. Hrsg. vom Wirtschafts- und sozialpolitischen Forschungs- und Bera- tungszentrum der Friedrich-Ebert-Stiftung, Bonn

Bundesagentur für Arbeit (BA) (2013): Der Arbeitsmarkt in Deutschland. Zeitarbeit in Deutschland - Aktuelle Entwicklungen. Arbeitsmarktberichterstattung Juli, Nürnberg, http://statistik.arbeitsagentur.de/Statischer-Content/Arbeitsmarktberichte/Arbeitsmarkt-Allgemein/generische-Publikationen/ Arbeitsmarkt-Deutschland-Zeitarbeit-Aktuelle-Entwicklung-2HJ2012.pdf Crimmann, A./Ziegler, K./Ellguth, P./Kohaut, S./Lehmer, F. (2009): Forschungsbericht zum Thema „Arbeitnehmerüberlassung“, Endbericht für das Bundesministerium für Arbeit und Soziales, Forschungsbericht Arbeitsmarkt (397),

Nürnberg, http://doku.iab.de/externe/2010/k100113303.pdf

Dörre K./Kraemer, K./Speidel, F. (2004): Prekäre Arbeit. Ursachen, soziale Auswirkungen und subjektive Verarbeitungsformen unsicherer Beschäftigungsverhältnisse, in: Das Argument (256), S. 378-397

Holst, H. (2009): Disziplinierung durch Leiharbeit? Neue Nutzungsstrategien von Leiharbeit und ihre arbeitspolitischen Folgen, in: WSI-Mitteilungen 62 (3), S. 143-149, http://www.boeckler.de/wsimit_2009_03_holst.pdf IG Metall (Hrsg.) (2008): Schwarz-Weiß-Buch Leiharbeit, Frankfurt a. M.

Jahn, E./Weber, E. (2013): Zusätzliche Jobs, aber auch Verdrängung, IAB-Kurzbericht 2/2013, http://doku.iab.de/kurzber/2013/kb0213.pdf

Keller, B./Seifert, H. (Hrsg.) (2007): Atypische Beschäftigung - Flexibilisierung und soziale Risiken, Berlin

Lehmer, F. (2012): Dient die Arbeitnehmerüberlassung für Langzeitarbeitslose als Brücke in nachhaltige Beschäftigung?, in: Sozialer Fortschritt 61 (8), S.190-197

Promberger, M. (2007): Leiharbeit - Flexibilität und Prekarität in der betrieblichen Praxis, in: Keller, B./Seifert, H. (Hrsg.): Atypische Beschäftigung. Flexibilisierung und soziale Risiken, Berlin, S. 127-144

Promberger, M. (2012): Topographie der Leiharbeit. Flexibilität und Prekarität einer atypischen Beschäftigungsform, Berlin

Schwitzer, H./Schumann, D. (2013): Tarifverträge über Branchenzuschläge eine erste Zwischenbilanz, in: WSI-Mitteilungen 66 (4), S.297-300, http://www.boeckler.de/wsimit_2013_04_schwitzer.pdf

Stenzel, C. (2012): Leiharbeit und betriebliche Mitbestimmung. Magisterarbeit an der Friedrich-Alexander-Universität Erlangen-Nürnberg, Erlangen, September 2012

Vogel, B. (Hrsg.) (2004): Leiharbeit. Neue sozialwissenschaftliche Befunde zu einer prekären Beschäftigungsform, Hamburg

Voss, E./Vitols, K. (2013): Leiharbeit in Europa: zwischen Flexibilität und Sicherheit, in: WSI-Mitteilungen 66 (8), S. 580-587, http://www.boeckler.de/ wsimit_2013_08_voss.pdf

Wassermann, W./Rudolph, W. (2007): Leiharbeit als Gegenstand betrieblicher Mitbestimmung: Hans-Böckler-Stiftung, Arbeitspapier (148), Düsseldorf Weinkopf, C./Vanselow, A. (2008): (Fehl-)Entwicklungen in der Zeitarbeit? Expertise im Auftrag der Friedrich-Ebert-Stiftung, Bonn

Wölfle, T. (2008): Gewerkschaftliche Strategien in der Leiharbeit, in: WSI Mitteilungen 61 (1), S. 38-44, http://www.boeckler.de/wsimit_2008_01_wolfle.pdf

\section{AUTORIN}

INGRID ARTUS, Dr., Professorin für Vergleichende Gesellschaftsanalyse am Institut für Soziologie der Friedrich-Alexander-Universität Erlangen-Nürnberg. Arbeitsschwerpunkte: Comparative Industrial Relations, Mitbestimmung, Arbeitskonflikte, Prekäre Arbeit.

ingrid.artus@fau.de 